\title{
Feasibility study of adjuvant chemotherapy with S-1 (TS-1; tegafur, gimeracil, oteracil potassium) for gastric cancer
}

\author{
Taira Kinoshita ${ }^{1}$, Atsushi Nashimoto ${ }^{2}$, Yoshitaka Yamamura ${ }^{3}$, Takeshi Okamura $^{4}$, Mitsuru Sasako $^{5}$, \\ Junichi Sakamoto ${ }^{6}$, Hiroshi Kojima ${ }^{6}$, Masahiro Hiratsuka ${ }^{7}$, Kuniyoshi Arai ${ }^{8}$, Motonori Sairenji, \\ Norimasa Fukushima ${ }^{10}$, Hironobu Kimura $^{11}$, and Toshifusa NaKajima ${ }^{12}$ \\ ${ }^{1}$ Department of Surgical Oncology, National Cancer Center Hospital East, 6-5-1 Kashiwanoha, Kashiwa, Chiba 277-8577, Japan \\ ${ }^{2}$ Niigata Cancer Center Hospital, Niigata, Japan \\ ${ }^{3}$ Aichi Cancer Center Hospital, Nagoya, Japan \\ ${ }^{4}$ National Kyushu Cancer Center, Fukuoka, Japan \\ ${ }^{5}$ National Cancer Center Hospital, Tokyo, Japan \\ ${ }^{6}$ Aichi Prefectural Hospital, Okazaki, Japan \\ ${ }^{7}$ Osaka Medical Center for Cancer and Cardiovascular Diseases, Osaka, Japan \\ ${ }^{8}$ Tokyo Metropolitan Komagome Hospital, Tokyo, Japan \\ ${ }^{9}$ Kanagawa Cancer Center, Yokohama, Japan \\ ${ }^{10}$ Yamagata Prefectural Central Hospital, Yamagata, Japan \\ ${ }^{11}$ Toyama Prefectural Central Hospital, Toyama, Japan \\ ${ }^{12}$ Cancer Institute Hospital, Tokyo, Japan
}

\begin{abstract}
Background. We conducted a feasibility study using S-1, a novel oral derivative of 5 -fluorouracil, as postoperative adjuvant chemotherapy for curatively resected gastric cancer patients.

Methods. Adjuvant chemotherapy consisted of eight courses (4-week administration and 2-week withdrawal) of S-1, at 80$120 \mathrm{mg} / \mathrm{body}$ per day. Forty-one patients from 11 institutions were enrolled in this pilot study, from November 1999 to October 2000.

Results. Thirty-five patients were eligible. In 7 patients, S-1 administration was discontinued due to recurrence. Among the 28 patients without recurrence, the planned eight courses of S-1 were administered to 17 patients $(60.7 \%)$. In 4 patients, S-1 administration was discontinued due to subjective symptoms, such as anorexia, in the first course. Adverse reactions such as neutropenia, leukopenia, elevated total bilirubin, anorexia, general fatigue, diarrhea, nausea, and stomatitis were seen in more than half of the patients. Although grade 3 neutropenia $(29.3 \%)$, leukopenia $(9.8 \%)$, and diarrhea $(9.8 \%)$ were observed, no grade 4 adverse effects appeared. Compared with the treatment of unresectable or recurrent gastric cancer with $\mathrm{S}-1$, the incidence of adverse reactions in the adjuvant setting was slightly higher, probably due to the influence of gastrectomy.

Conclusion. Except for the early development of anorexia, most likely due to adverse effects of surgery, postoperative administration of S-1 for 1 year seems feasible as adjuvant chemotherapy for gastric cancer.
\end{abstract}

Offprint requests to: T. Kinoshita

Received: November 5, 2003 / Accepted: February 23, 2004
Key words Gastric cancer · S-1 · Adjuvant chemotherapy · Feasibility study

\section{Introduction}

The results of surgical treatment for gastric cancer have been improved by early detection and meticulous surgical procedures in Japan. However, we still face recurrence in patients with advanced gastric cancer, even with extended surgical treatment [1].

Many clinical trials of adjuvant chemotherapy could not prove survival benefits. Only a few metaanalyses of adjuvant trials reported the possibility of survival benefits [2,3]. According to the guidelines from the Japanese Gastric Cancer Association [4], the efficacy of adjuvant chemotherapy after curative resection for gastric cancer has not been established. A well-designed large-scale phase III trial is needed, with a surgeryalone arm, to prove the benefit of adjuvant chemotherapy.

$\mathrm{S}-1$ is a dihydropyrimidine dehydrogenase (DPD)inhibitory fluoropyrimidine (DIF), which showed the highest response rate among many oral anticancer agents against unresectable advanced gastric cancer in early and late phase II studies [5-7]. In these phase II trials, S-1 showed $40 \%$ and higher response rates with acceptable low toxicity. Based on these results, a phase III trial to compare $\mathrm{S}-1$ with two other regimens is underway as a JCOG (Japan Clinical Oncology Group) trial for unresectable and recurrent gastric cancer. 
At the same time, the high response rate and low toxicity of S-1 has led to its adjuvant use. Oral anticancer drugs are also attractive for outpatient use. A feasibility study to confirm the safety of S-1 for adjuvant chemotherapy after curative surgery was necessary before starting the phase III trial.

\section{Patients and methods}

\section{Design of the trial}

The trial was non-blinded and open-label. The primary endpoint was the rate of accomplishment of the scheduled adjuvant chemotherapy. Secondary endpoints were the incidence and grade of adverse reactions.

In this trial, the sample size was 50, without any calculations based on statistical assumptions.

\section{Patient eligibility}

Patient eligibility required compliance with the following criteria: gastric adenocarcinoma with histological proof; operative findings revealing advanced gastric cancer (T2 or more); curability B resection, defined in the Japanese classification of gastric carcinoma [8]; sufficient oral intake; no prior treatment except for surgery; and more than 19 and less than 76 years of age. Patients also had to have adequate organ function $(4000 \leq$ leukocytes $<12000 / \mathrm{mm}^{3}$; thrombocytes, $\geq 100000 / \mathrm{mm}^{3}$; total bilirubin, $\leq 1.5 \mathrm{mg} / \mathrm{dl}$; GOT and GPT, less than twice the normal limits at each institution; creatinine, $\leq 1.5 \mathrm{mg} / \mathrm{dl}$ ). Patients expected to receive medication and to be followed-up regularly for more than 48 weeks. Patients with a history of drug hypersensitivity, serious surgical and non-surgical complications, or active secondary cancer were excluded. Pregnant or lactating women were excluded. This study was approved by the institutional review board at each site, and written informed consent was obtained from all patients.

\section{Treatment schedule}

Chemotherapy consisted of eight courses (4-week administration and 2-week withdrawal) of S-1 (tegafur, gineracil, oteracil potassium; Taiho Pharmocentical, Tokyo, Japan) at $80-120 \mathrm{mg} /$ body per day according to the body surface area (BSA): BSA $<1.25 \mathrm{~m}^{2}, 80 \mathrm{mg} /$ day; $1.25 \leq$ BSA $<1.5 \mathrm{~m}^{2}, 100 \mathrm{mg} /$ day; $1.5 \mathrm{~m}^{2} \leq \mathrm{BSA}, 120 \mathrm{mg} /$ day. S-1 was administered orally, twice daily after meals for 4 weeks after surgery. Doses were modified in accordance with the following guidelines. When adverse reactions appeared, the dose was reduced from 120 to $100 \mathrm{mg} /$ day or from 100 to $80 \mathrm{mg} /$ day, or administration was temporarily discontinued. The withdrawal period due to adverse reactions was less than 16 days in the same course, with a maximum of 28 days' administration of the drugs in total. Treatment was discontinued when the patient showed recurrence of disease or adverse reactions that were uncontrollable by dose modification and temporary withdrawal of drug administration. A 48-week period of temporary drug withdrawal was the limit set for a patient not being able to enter a new course.

\section{Evaluation of toxicity}

The National Cancer Institute Common Toxicity Criteria (NCI-CTC; 1998) were adopted to determine the toxicity of the chemotherapy.

\section{Results}

In November 2000, an interim analysis was performed in order to determine whether to continue further recruitment of patients. Because information from the periodic safety report of use-results surveillance (September 24, 2000) from Taiho Pharmaceutical to the Ministry of Health, Labor, and Welfare revealed there were no specific adverse reactions in up to two courses in 110 patients who received S-1 within 30 days after surgical resection of gastric cancer, and because a feasible result was obtained by monitoring the patients in the present study, we decided to complete recruitment at 41 patients.

Of the 41 patients, 6 patients were ineligible. Four patients had received curable A resection, 1 patient had received intraoperative chemotherapy with cisplatin (CDDP), and the other patient had distant metastasis at the time of the operation. Thirty-five patients were eligible (full analysis set [FAS]).

Table 1 shows the characteristics of the FAS. Thirty of the 35 patients $(85.7 \%)$ had rather advanced stage disease (TNM stage IIIA or more; Table 1).

Table 2 shows drug compliance in each course and the reasons for discontinuation of drug administration. In 7 of the 35 patients (FAS), administration of S-1 was discontinued due to recurrence. The planned eight courses of S-1 were administered to 17 patients (60.7\%). In 4 patients, drug administration was discontinued in the first course at the patient's request, due to anorexia. The main reason for discontinuation was recurrence of disease.

Table 3 shows the drug compliance of the FAS (days and total amount of the drug). In every course, drug compliance was maintained at more than $85 \%(86.0 \%-$ $90.4 \%$ ). In the total of 35 patients (FAS), the percentage of actual administration days against the total number of planned administration days (28 days $\times 8$; 
Table 1. Patient characteristics

\begin{tabular}{|c|c|c|c|}
\hline & & Number of patients & Percentage \\
\hline \multirow{2}{*}{ Sex } & Male & 20 & 57.1 \\
\hline & Female & 15 & 42.9 \\
\hline \multirow[t]{7}{*}{ Age (years) } & $20-29$ & 1 & 2.9 \\
\hline & $30-39$ & 2 & 5.7 \\
\hline & $40-49$ & 5 & 14.3 \\
\hline & $50-59$ & 4 & 11.4 \\
\hline & $60-69$ & 12 & 34.3 \\
\hline & $70-79$ & 11 & 31.4 \\
\hline & Mean, 60.3 ; median, 65.0 & & \\
\hline \multirow[t]{4}{*}{$\operatorname{BSA}\left(\mathrm{m}^{2}\right)$} & $1.20-1.39$ & 10 & 28.6 \\
\hline & $1.40-1.59$ & 20 & 57.1 \\
\hline & $1.60-1.79$ & 5 & 14.3 \\
\hline & Mean, $1.47 ;$ median 1.47 & & \\
\hline \multirow[t]{2}{*}{ Lymph node dissection } & $\mathrm{D} 2$ & 24 & 68.6 \\
\hline & D3 & 11 & 31.4 \\
\hline \multirow[t]{3}{*}{ Type of resection } & Distal gastrectomy & 15 & 42.9 \\
\hline & Total gastrectomy & 19 & 54.3 \\
\hline & Proximal gastrectomy & 1 & 2.9 \\
\hline \multirow[t]{2}{*}{ Combined resection } & No & 11 & 31.4 \\
\hline & Yes & 24 & 68.6 \\
\hline \multirow[t]{5}{*}{ Reconstruction } & Billroth I & 5 & 14.3 \\
\hline & Billroth II & 8 & 22.9 \\
\hline & Roux-Y & 20 & 57.1 \\
\hline & Interposition & 1 & 2.9 \\
\hline & Other & 1 & 2.9 \\
\hline \multirow[t]{5}{*}{ Japanese Stage } & IB & 1 & 2.9 \\
\hline & II & 3 & 8.6 \\
\hline & IIIA & 12 & 34.3 \\
\hline & IIIB & 9 & 25.7 \\
\hline & IV & 10 & 28.6 \\
\hline \multirow[t]{5}{*}{ TNM Stage } & IB & 2 & 5.7 \\
\hline & II & 3 & 8.6 \\
\hline & IIIA & 9 & 25.7 \\
\hline & IIIB & 8 & 22.9 \\
\hline & IV & 13 & 37.1 \\
\hline
\end{tabular}

BSA, body surface area

224) was $79.0 \%$ (median) and $69.7 \%$ (mean). Concerning the amount of the drug, compliance was $75.2 \%$ (median) and 67.3\% (mean).

Table 4 shows summaries of the adverse reactions that developed in more than $10 \%$ of the 41 patients in total, grouped as laboratory findings-based and clinical findings-based. Of the laboratory findings-based adverse reactions, neutropenia was the most frequent, in 35 of the 41 patients $(85.4 \%)$ followed by leukopenia $(75.6 \%)$, increase in serum total bilirubin $(53.7 \%)$, GOT (41.5\%), anemia (hemoglobin [Hb]; 41.5\%), anemia (RBC; 34.1\%), alkaline phosphatase (ALP; $26.8 \%$ ), GPT $(26.8 \%)$, lactate dehydrog-enase (LDH; $24.4 \%)$, thrombocytopenia $(24.4 \%)$, proteinuria $(24.4 \%)$, lymphopenia $(19.5 \%)$, anemia (hematocrit [Hct]; 14.6\%), hyperkalemia (12.2\%), blood urea nitrogen (BUN; 12.2\%), and hypoalbuminemia (12.2\%).
Among the clinical findings-based adverse reactions, anorexia was the most frequent $(68.3 \%)$, followed by fatigue $(61.0 \%)$, diarrhea $(58.5 \%)$, nausea $(51.2 \%)$, stomatitis $(51.2 \%)$, pigmentation changes $(46.3 \%)$, weight loss $(39.0 \%)$, rash $(31.7 \%)$, and vomiting (19.5\%). Concerning the incidence and grade of laboratory findings-based adverse reactions, grade 3 adverse reactions were seen with neutropenia, leucopenia, lymphopenia, anemia ( $\mathrm{Hb})$, GOT, and GPT. However, there were no grade 4 adverse reactions. In the clinical findings-based adverse reactions, grade 3 adverse reactions were observed with anorexia, fatigue, diarrhea, and weight loss. There were also no grade 4 adverse reactions.

Figure 1 shows comparisons of the incidences of the main adverse reactions in a late-phase II study [9] and those in the present study. When compared with the 
Table 2. Drug compliance (each course)

\begin{tabular}{|c|c|c|c|c|c|}
\hline \multirow[b]{2}{*}{$\begin{array}{l}\text { Course } \\
\text { no. }\end{array}$} & \multicolumn{2}{|c|}{$\begin{array}{l}\text { FAS full analysis set; } \\
\qquad n=35\end{array}$} & \multicolumn{2}{|c|}{$\begin{array}{l}\text { Excluding patients with } \\
\text { recurrence }(n=28)\end{array}$} & \multirow{2}{*}{$\begin{array}{l}\text { Reasons for } \\
\text { discontinuation of drug } \\
\text { administration }\end{array}$} \\
\hline & $\begin{array}{l}\text { Number of patients } \\
\text { entering the course }\end{array}$ & Percentage & $\begin{array}{l}\text { Number of patients } \\
\text { entering the course }\end{array}$ & Percentage & \\
\hline 1 & 35 & - & 28 & - & $\begin{array}{l}\text { Patient refusal } \\
\quad(\text { anorexia; } n=4) \\
\text { Complication, } \\
\quad(\text { varicose; } n=1)\end{array}$ \\
\hline 2 & 30 & 85.7 & 23 & 82.1 & \\
\hline 3 & 30 & 85.7 & 23 & 82.1 & Recurrence $(n=1)$ \\
\hline 4 & 29 & 82.9 & 23 & 82.1 & $\begin{array}{l}\text { Patient refusal } \\
\quad(\text { anorexia; } n=1) \\
\text { Dr's judgment } \\
\quad \text { (poor general } \\
\quad \text { condition; } n=1)\end{array}$ \\
\hline 5 & 27 & 77.1 & 21 & 75.0 & Recurrence $(n=3)$ \\
\hline 6 & 24 & 68.6 & 21 & 75.0 & $\begin{array}{l}\text { Recurrence }(n=2) \\
\text { Adverse reaction } \\
\quad(\text { arrhythmia; } n=1)\end{array}$ \\
\hline 7 & 21 & 60.0 & 20 & 71.4 & $\begin{array}{l}\text { Recurrence }(n=1) \\
\text { Unable to enter the } \\
\quad \text { eight course }(n=3)^{\mathrm{a}}\end{array}$ \\
\hline 8 & 17 & 48.6 & 17 & 60.7 & $\begin{array}{l}\text { Patient refusal } \\
\quad(\text { adverse reaction; } n=1)\end{array}$ \\
\hline
\end{tabular}

${ }^{a}$ Because of prolongation of the period during which the drug was temporarily withdrawn

Table 3. Drug compliance (days and total amount of the drug)

\begin{tabular}{lccc}
\hline Course no. & $\begin{array}{c}\text { No. of patients } \\
\text { entering the course }\end{array}$ & $\begin{array}{c}\text { Percent administration } \\
\text { days (mean) }\end{array}$ & $\begin{array}{c}\text { Percent administration } \\
\text { amount (mean) }\end{array}$ \\
\hline 1 & 35 & 85.9 & 87.9 \\
2 & 30 & 91.4 & 90.4 \\
3 & 30 & 91.7 & 88.1 \\
4 & 29 & 92.3 & 87.4 \\
5 & 27 & 96.1 & 90.4 \\
6 & 24 & 92.1 & 89.0 \\
7 & 21 & 93.7 & 86.0 \\
8 & 17 & 91.8 & 87.1 \\
Overall mean $(n=35)$ & 69.7 & 67.3 \\
Overall median $(n=35)$ & 79.0 & 75.2 \\
\hline
\end{tabular}

${ }^{a}$ Days actually administered as a percentage of planned number of days

${ }^{\mathrm{b}}$ Amount of drug as actually administered a percentage of planned amount

data from the late-phase II study [9], a higher incidence of adverse reactions was observed in the present study.

\section{Discussion}

As mentioned in the "Introduction", S-1 is an attractive oral anticancer agent for advanced gastric cancer, with a high response rate and low toxicity. The possibility of outpatient use of S-1 has increased the convenience for both doctors and patients, and it has led to the idea of using S-1 as an adjuvant chemotherapeutic agent. Up to 1999 , there were no trials of S-1 use in the adjuvant setting. Therefore, as a prerequisite to conducting a large-scale clinical trial of adjuvant $\mathrm{S}-1$, the present study was carried out to confirm the feasibility of adjuvant S-1 given after curative gastrectomy.

Survival benefits of adjuvant chemotherapy after curative resection of gastric cancer have not yet been proved by a large-scale prospective randomized trial, as stated in the guidelines of the Japanese Gastric Cancer Association (Japanese guidelines). Even though 
Table 4. Adverse reactions $(n=41)$

\begin{tabular}{ccccc}
\multicolumn{9}{c}{ Grade } & \\
\hline & 3 & 2 & 1 & (incidence; percentage)
\end{tabular}

\begin{tabular}{|c|c|c|c|c|}
\hline \multicolumn{5}{|l|}{ Laboratory findings } \\
\hline Neutropenia & 12 & 16 & 7 & 85.4 \\
\hline Leukopenia & 4 & 15 & 12 & 75.6 \\
\hline Lymphopenia & 2 & 5 & 1 & 19.5 \\
\hline Thrombocytopenia & & 2 & 8 & 24.4 \\
\hline Anemia $(\mathrm{Hb})$ & 3 & 8 & 6 & 41.5 \\
\hline Anemia (RBC) & & 5 & 9 & 34.1 \\
\hline Anemia (Hct) & & 3 & 3 & 14.6 \\
\hline GOT & 2 & 1 & 14 & 41.5 \\
\hline GPT & 1 & 2 & 8 & 26.8 \\
\hline $\mathrm{LDH}$ & & & 10 & 24.4 \\
\hline ALP & & 1 & 10 & 26.8 \\
\hline Total bilirubin & & 8 & 14 & 53.7 \\
\hline Hypoalbuminemia & & 1 & 4 & 12.2 \\
\hline Hyperkalemia & & & 5 & 12.2 \\
\hline BUN & & & 5 & 12.2 \\
\hline Proteinuria & & 1 & 9 & 24.4 \\
\hline \multicolumn{5}{|l|}{ Clinical findings } \\
\hline Anorexia & 4 & 5 & 19 & 68.3 \\
\hline Nausea & & 3 & 18 & 51.2 \\
\hline Vomiting & & & 8 & 19.5 \\
\hline Diarrhea & 4 & 5 & 15 & 58.5 \\
\hline Stomatitis & & 2 & 19 & 51.2 \\
\hline Fatigue & 1 & 5 & 19 & 61.0 \\
\hline Pigmentation changes & & 3 & 16 & 46.3 \\
\hline Rash & & 5 & 8 & 31.7 \\
\hline Weight loss & 1 & 9 & 6 & 39.0 \\
\hline
\end{tabular}

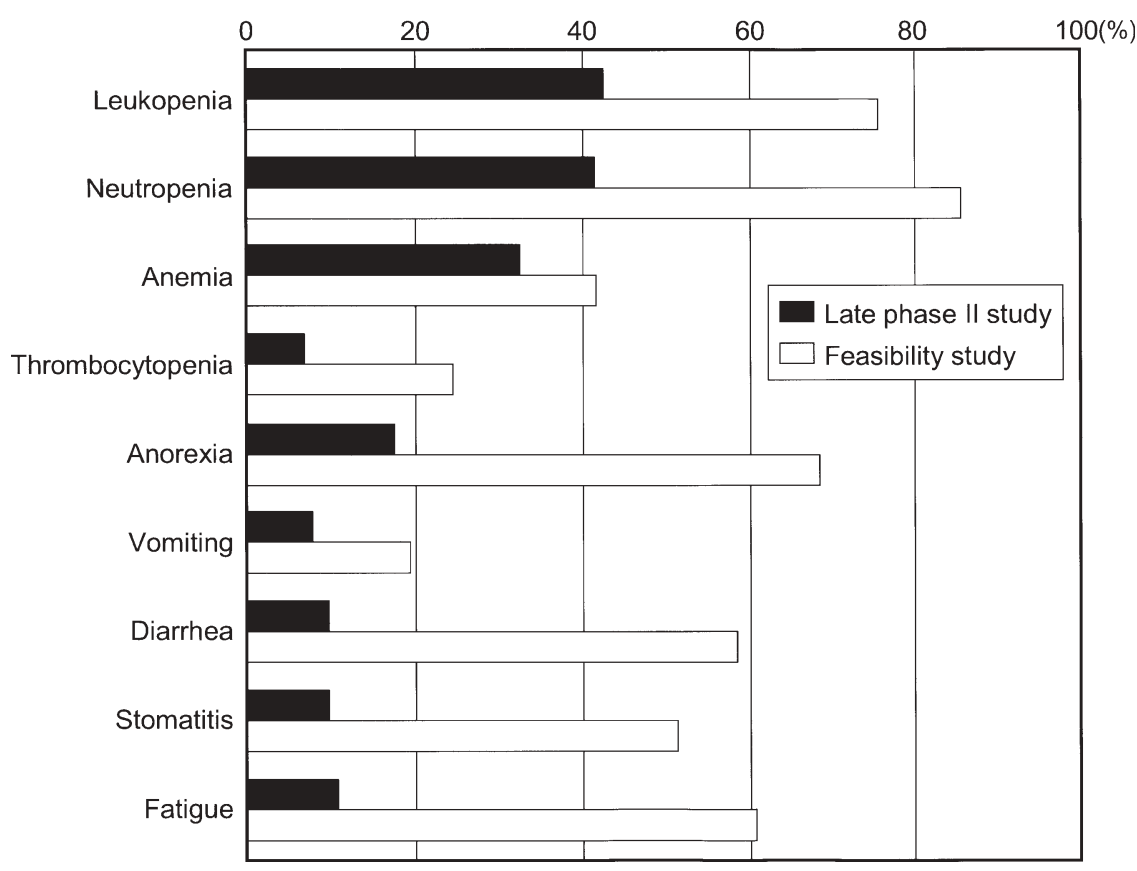

Fig. 1. Comparison of incidence of adverse reactions with that in a late phase II study [9] 
Macdonald et al. [9], in 2001, confirmed the effectiveness of the combination of 5-fluorouracil with radiotherapy as an adjuvant after curative gastric surgery in a randomized clinical trial, the Japanese guidelines have not changed up to now, probably because of differences in the local control rates after different types of surgery between the United States and Japan. The local recurrence rate is negligible after systematic lymph node dissection (D2 or more) in Japan. However, in the United States, the local recurrence rate is usually much higher with less aggressive surgery (D0 or D1). The incidence of local, invisible, residual cancer cells in eligible patients in the study of Macdonald et al. [9] were quite different from those in the Japanese candidates for adjuvant therapy. That is why we still think that survival benefits of adjuvant chemotherapy after curative resection of gastric cancer have not yet been proved in Japan; only metaanalyses will show the survival benefit of adjuvant chemotherapy.

$\mathrm{S}-1$ is expected to be a promising agent for adjuvant use. The high response rate of S-1 in advanced gastric cancer gives a rationale for expecting a certain survival benefit with S-1 in the adjuvant setting.

Because the standard treatment of locally advanced gastric cancer is still surgery, a randomized controlled trial with a surgery-alone arm is essential to prove the efficacy of adjuvant chemotherapy.

According to the "Results", no grade 4 adverse reactions were observed.

In the FAS, excluding the patients with recurrence, 17 of the 28 patients $(60.7 \%)$ received the planned eight courses of S-1. Drug compliance was acceptable. In every course, drug compliance was over $85 \%$ in the FAS.

Problems in this study were a higher incidence of adverse reactions when compared with that in the phase II trials and postmarketing surveillance [10], and a high incidence of patient refusal, due to adverse reactions in the first course, which was not seen during the postmarketing surveillance of 110 patients who received S-1 within 30 days after surgical resection of gastric cancer (as mentioned in "results"). The reasons for these problems, especially the early appearance of anorexia, may be the influence of surgery, because the patients in this study had rather advanced disease and had received D2 or more aggressive gastrectomy with frequent combined organ resections. It is difficult to deny the possibility of gastro-intestinal (GI) toxicity of S-1; however, the adverse reaction was not bone marrow suppression, which is a dose-limiting toxicity of S-1. In this protocol, S-1 administration was started within 4 weeks after surgery. In the early postoperative period, patients have not yet recovered from surgical stress, and the limitation of food intake due to aggressive gastrectomy is a possible cause of exacerbation of adverse reactions such as anorexia and nausea. To prevent these problems, a delay in the start of drug administration seems necessary for adjuvant use. Except for these problems, the administration of S-1 for 1 year seems feasible as postoperative adjuvant chemotherapy for gastric cancer.

Based on this feasibility study, a prospective randomized controlled trial was started in 2001 to evaluate the efficacy of S-1 as adjuvant chemotherapy; in this trial S1 administration is started within 6 weeks after surgery.

We expect that a significant survival benefit of S-1, with less toxicity, will be shown by this trial, and that this could be the standard treatment after curative gastrectomy.

\section{References}

1. Maruyama K, Okabayashi K, Kinoshita T. Progress in gastric cancer surgery in Japan and its limits of radicality. World J Surg 1987;11:418-25.

2. Nakajima T, Ohta K, Ohyama S, Hamashima N. Meta-analysis of adjuvant chemotherapy trial for gastric cancer at the Cancer Institute Hospital, Tokyo. In: Nakajima T, Yamaguchi T. editors. Multimodality therapy for gastric cancer. Heidelberg Berlin New York Tokyo: Springer-Verlag; 1988. pp. 27-31.

3. Hermans J, Bonenkamp JJ, Boon MC, Bunt AM, Ohyama S, Sasako M, et al. Adjuvant therapy after curative resection for gastric cancer: meta-analysis of randomized trials. J Clin Oncol 1993;11:1441-47.

4. Nakajima T. Gastric cancer treatment guidelines in Japan. Gastric Cancer 2002;5:1-5.

5. Sugimachi K, Maehara Y, Horikoshi N, Shimada Y, Sakata Y, Miyachi Y, et al. An early phase II study of oral S-1, a newly developed 5-fluorouracil derivative for advanced and recurrent gastrointestinal cancers. Oncology 1999;57:202-10.

6. Sakata Y, Ohtsu A, Horikoshi N, Sugimachi K, Mitachi Y, Taguchi T. Late phase II study of novel oral fluoropyrimidine anticancer drug S-1 (1 M tegafur-04 M gimestat-1 M otastat potassium) in advanced gastric cancer patients. Eur J Cancer 1998;34: 1715-20.

7. Koizumi W, Kurihara M, Nakano S, Hasegawa K. Phase II study of S-1, a novel oral derivative of 5-fluorouracil, in advanced gastric cancer. Oncology 2000;58:191-7.

8. Japanese Gastric Cancer Association. Japanese classification of gastric carcinoma. 2nd English Ed. Gastric Cancer 1998;1:1024.

9. Macdonald JS, Smalley SR, Benedetti J, Hundahl SA, Estes NC, Stemmermann GN, et al. Chemoradiotherapy after surgery compared with surgery alone for adenocarcinoma of the stomach or gastroesophageal junction. N Engl J Med 2001;345:725-30.

10. Maehara Y.S-1 in gastric cancer: a comprehensive review. Gastric Cancer 2003;6 (Suppl 1):2-8. 